\title{
PROBABILIDADE DE OCORRÊNCIA DE VALORES ESTIMADOS DE EVAPOTRANSPIRAÇÃO DE REFERÊNCIA NO SERTÃO DO CEARÁ
}

\author{
Mairton Gomes da Silva ${ }^{1}$, Lucas dos Santos Batista ${ }^{2}$, Francicleiton Freires do Carmo ${ }^{3}$, Nara Tosta Santos ${ }^{4}$, Itamar de \\ Souza Oliveira ${ }^{5}$
}

\section{RESUMO}

Devido à grande variação nos elementos meteorológicos nas diferentes regiões, provocando, com isso, dispersão nas estimativas dos valores de evapotranspiração de referência $\left(\mathrm{ET}_{\mathrm{o}}\right)$, torna-se necessária o estudo de funções de distribuições de probabilidade para um correto dimensionamento dos sistemas de irrigação. Diante do contexto, neste trabalho, objetivou-se avaliar o ajuste dos valores de $\mathrm{ET}_{\mathrm{o}}$ mensal utilizando o método FAO-Penman-Monteith, às distribuições de probabilidade: Normal, Log-Normal e Gama para os municípios de Crateús, Quixeramobim e Tauá, no estado do Ceará. Para verificar a aderência das distribuições empíricas à distribuição teórica foram utilizados os testes de Kolmogorov-Smirnov (KS) e Qui-quadrado $\left(\chi^{2}\right)$, em nível de 5\% de significância. As distribuições de probabilidade Normal, Log-Normal e Gama mostraram-se adequadas para representar a ET nos municípios de Crateús, Quixeramobim e Tauá, em todos os meses do ano, segundo o teste KS. Ressalta-se a fácil aplicação da distribuição Normal, bem como a obtenção de seus parâmetros.

Palavras-chave: distribuições de probabilidade, Penman-Monteith, testes de aderência

\section{ABSTRACT \\ PROBABILITY OF OCCURRENCE OF ESTIMATED VALUES FOR REFERENCE EVAPOTRANSPIRATION IN THE BACKLANDS OF CEARÁ, BRAZIL}

Due to the great variation of the meteorological elements in different regions, thus causing dispersion in the estimates of the values of reference evapotranspiration $\left(\mathrm{ET}_{\mathrm{o}}\right)$, it is necessary the study functions of probability distributions for the correct dimensioning of irrigation systems. Given the context, this work aimed to evaluate the adjustment of monthly values of $\mathrm{ET}_{\mathrm{o}}$ using the FAO-Penman-Monteith method to the probability distributions: Normal, LogNormal and Gamma for the municipalities Crateús, Quixeramobim and Tauá in the state of Ceará, Brazil. To verify the adherence of empirical distributions to theoretical distribution the Kolmogorov-Smirnov (KS) and Chi-square $\left(\chi^{2}\right)$ tests were used, as the significance level of 5\%. The probability distributions Normal, Log-Normal and Gamma showed to be adequate to represent the ET in the municipalities of Crateús, Quixeramobim and Tauá in all months of the year, according to the KS test. It is important to highlight the easy application of the Normal distribution, as well as acquisition of its parameters.

Keywords: probability distributions, Penman-Monteith, adherence tests

\section{Recebido para publicação em 18/08/2013. Aprovado em 09/10/2014.}

1 - Tecnólogo em Irrigação e Drenagem, Mestre em Engenharia Agrícola - UFRB, Cruz das Almas-BA, e-mail: mairtong@hotmail.com

2 - Graduando em Engenharia Agronômica - UFRB, Cruz das Almas-BA, e-mail: lucasbaptistaufrb@gmail.com

3 - Tecnólogo em Irrigação e Drenagem, Mestre em Irrigação e Drenagem, Doutorando em Engenharia Agrícola - UFRB, Cruz das Almas-BA, e-mail: cleitontid@hotmail.com

4- Engenheira Agrônoma, Mestranda em Engenharia Agrícola - UFRB, Cruz das Almas-BA, e-mail: narinhatosta@ @hotmail.com

5- Graduando em Engenharia Agronômica - UFRB, Cruz das Almas-BA, e-mail: itamar25souza@gmail.com 


\section{INTRODUÇÃO}

A grande variabilidade temporal e espacial da distribuição das chuvas, principalmente em regiões áridas e semiáridas, determina altos níveis de risco às atividades agrícolas. Nesse contexto, a irrigação é uma alternativa utilizada para viabilizar e aumentar a produção agrícola em locais que estejam sujeitos a baixos índices pluviométricos (SANTOS et al., 2011).

A evapotranspiração de referência (ETó $)$ é um parâmetro utilizado para diferentes fins, como nas estimativas dos balanços hídricos agrícolas e na modelagem dos processos climatológicos e hidrológicos, tendo como a finalidade de se obter as estimativas das necessidades de irrigação, previsão de safras, avaliação da disponibilidade dos recursos hídricos e na caracterização climática de uma determinada região.

A probabilidade de ocorrência de valores estimados de evapotranspiração é importante instrumento na tomada de decisões relacionadas às atividades agropecuárias (PRELA-PANTANO et al., 2010). Nos projetos de irrigação, o critério de escolha do nível de probabilidade deve estar baseado em uma análise econômica, considerando-se os prejuízos associados à redução da quantidade e da qualidade da produção, decorrentes da deficiência hídrica, e o aumento de custos do sistema para satisfazer níveis mais elevados de probabilidade (SILVA et al., 1998). $\mathrm{O}$ valor de $\mathrm{ET}_{\mathrm{o}}$ com $75 \%$ de probabilidade tem sido recomendado para o dimensionamento de projetos de irrigação (BACK, 2007).

O estudo de distribuições de probabilidade de variáveis climáticas ao longo do tempo, como forma de compreender os fenômenos meteorológicos para determinar seus padrões de ocorrência e previsões probabilísticas, permitem ao pesquisador uma previsibilidade razoável do comportamento climático de uma região, sendo este fato, uma ferramenta de grande importância para o planejamento e gestão de diversas atividades agrícolas e humanas (ASSIS et al., 2013).

Trabalhos de ajustes de distribuição de probabilidade usando função de distribuição de probabilidade teóricas em relação às variáveis climáticas, como evapotranspiração (SILVA et al., 1998; SAAD et al., 2002; ABUMANSSUR, 2006; BLAIN; BRUNINI, 2007; ALMEIDA et al., 2009; CAVALCANTE JUNIOR et al., 2009; BACELAR et al., 2011; PEIXOTO et al., 2011a,b; JERSZURKI et al., 2012; VELLAME et al., 2012; ASSIS;
SOUSA, 2013; BORGES JÚNIOR et al., 2013; SILVA et al., 2013a; ASSIS et al., 2014; SANTOS et al., 2014; SILVA et al., 2014a,b; SOUZA et al., 2014) foram desenvolvidos enfatizando os benefícios no planejamento de atividades que reduzem prováveis riscos climáticos.

Com o trabalho, objetivou-se verificar o ajuste de valores mensais estimados de evapotranspiração de referência $\left(E T_{0}\right)$ utilizando o método de FAO-Penman-Monteith, as distribuições de probabilidade: Normal, Log-Normal e Gama nos municípios de Crateús, Quixeramobim e Tauá no estado do Ceará, Brasil.

\section{MATERIAL E MÉTODOS}

O trabalho foi realizado a partir das médias mensais de temperatura máxima e mínima do ar, umidade relativa do ar, velocidade do vento e insolação, oriundos de estações convencionais dos municípios de Crateús (5'10’42” S, 4040’39” W e altitude de $274,70 \mathrm{~m}$ ), Quixeramobim ( $5^{\circ} 11^{\prime} 57^{\prime}$ " S, 39¹7'34" W e altitude de 191,7 m) e Tauá (600'11' S, 40¹7'34" W e altitude de 402,7 m), compreendendo o período de 1961 a 2011. Os dados foram obtidos do Instituto Nacional de Meteorologia (INMET), a partir Banco de Dados Meteorológicos para Ensino e Pesquisa (BDMEP) (Figura 1).

O município de Crateús está situado a oeste do estado na microrregião geográfica do Sertão de Crateús e pertencente à mesorregião dos Sertões cearenses. Oclima da região, segundo a classificação de Köppen é do tipo Aw', é quente com temperaturas máximas em outubro, temperatura média anual de $26{ }^{\circ} \mathrm{C}$ a $28^{\circ} \mathrm{C}$ e precipitação média anual de 731,2 $\mathrm{mm}$, concentrada no período de janeiro a abril. O município de Quixeramobim está situado na parte central do estado na microrregião geográfica do Sertão de Quixeramobim e pertencente à mesorregião dos Sertões cearenses. O clima é do tipo tropical quente semiárido, enquadrando-se na classificação climática de Köppen como BShw'. A precipitação pluviométrica média anual é de 707,7 $\mathrm{mm}$ e temperatura média anual de $26^{\circ} \mathrm{C}$ a $28^{\circ} \mathrm{C}$. O município de Tauá está situado a sudoeste do estado na microrregião geográfica do Sertão dos Inhamuns e pertencente à mesorregião dos Sertões cearenses. Segundo classificação de Köppen, o clima é do tipo BSw'h', clima quente e semiárido, com temperatura superior a $18^{\circ} \mathrm{C}$ no mês mais frio e precipitação pluviométrica média anual de 550 a $650 \mathrm{~mm}$ (SILVA et al., 2013b). 


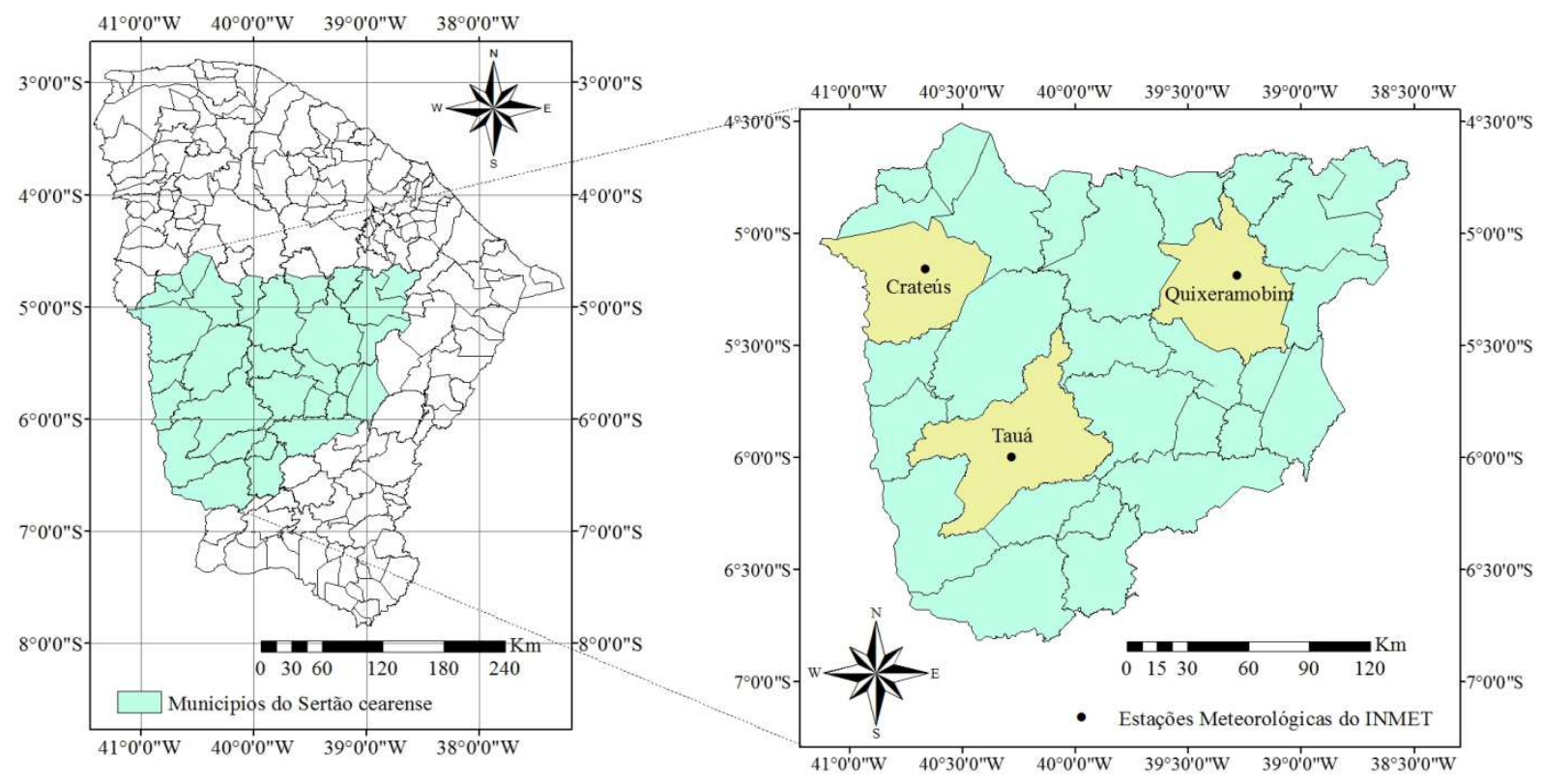

(Fonte: SILVA et al., 2013).

Figura 1. Localização geográfica das estações meteorológicas utilizadas neste estudo, no sertão do estado do Ceará, Brasil.

A evapotranspiração de referência foi estimativa pelo método de Penman-Monteith, parametrizado pela FAO no seu manual número 56 (ALLEN et al., 1998), Equação 1.

$$
\mathrm{ET}_{\mathrm{o}}=\frac{0,408 \Delta\left(\mathrm{R}_{\mathrm{n}}-\mathrm{G}\right)+\gamma \frac{900}{\mathrm{~T}_{\mathrm{m}}+273} \mathrm{u}_{2}\left(\mathrm{e}_{\mathrm{s}}-\mathrm{e}_{\mathrm{a}}\right)}{\Delta+\gamma\left(1+0,34 \cdot \mathrm{u}_{2}\right)}
$$

em que,

$\mathrm{ET}_{\mathrm{o}}=$ evapotranspiração de referência, $\mathrm{mm} \mathrm{dia}^{-1}$; $\mathrm{R}_{\mathrm{n}}=$ radiação líquida total do gramado, $\mathrm{MJ} \mathrm{m}^{-2} \mathrm{dia}^{-1}$; $\mathrm{G}=$ densidade do fluxo de calor no solo, $\mathrm{MJ} \mathrm{m}^{-2} \mathrm{dia}^{-1}$; $\mathrm{T}_{\mathrm{m}}=$ temperatura média diária do $\mathrm{ar},{ }^{\circ} \mathrm{C}$;

$\mathrm{u}_{2}=$ velocidade do vento média diária a $2 \mathrm{~m}$ de altura, $\mathrm{m} \mathrm{s}^{-1}$;

$\mathrm{e}_{\mathrm{s}}=$ pressão de saturação de vapor, $\mathrm{kPa}$;

$\mathrm{e}_{\mathrm{a}}=$ pressão parcial de vapor, $\mathrm{kPa}$;

$\mathrm{e}_{\mathrm{s}}-\mathrm{e}_{\mathrm{a}}=$ déficit de saturação de vapor, $\mathrm{kPa}$;

$\Delta=$ declividade da curva de pressão de vapor no ponto de $\mathrm{T}_{\mathrm{m}}, \mathrm{kPa}^{\circ} \mathrm{C}^{-1}$; e

$\gamma=$ coeficiente psicrométrico, $\mathrm{kPa}^{\circ} \mathrm{C}^{-1}$.

A distribuição Normal consiste de uma distribuição de dois parâmetros, que tem sua função densidade de probabilidade da seguinte forma
(CATALUNHA et al., 2002; LYRA et al., 2006; JUNQUEIRA JÚNIOR et al., 2007), Equação 2.

$F(x)=\frac{1}{\sigma \sqrt{2 \pi}} e\left(-\frac{(x-\mu)^{2}}{2 \sigma^{2}}\right) ;$ para $-\infty<x<+\infty$

em que,

$\mathrm{x}=$ valor da variável aleatória;

$\mu=$ média da variável $\mathrm{x}$; e

$\sigma=$ desvio-padrão da variável $\mathrm{x}$.

A função densidade da distribuição LogNormal a dois parâmetros e a três parâmetros são representadas da seguinte forma (CATALUNHA et al., 2002; RIBEIRO et al., 2007), Equação 3.

$F(x)=\frac{1}{(x-a) \sigma \sqrt{2 \pi}} e\left(-\frac{\ln [(x-a)-\mu]^{2}}{2 \sigma^{2}}\right)$

em que,

$\mathrm{x}=$ valor da variável aleatória;

$\mu=$ média dos logaritmos da variável $\mathrm{x}$;

$\sigma=$ desvio-padrão dos logaritmos da variável x; e $\mathrm{a}=$ limite inferior da amostra. 
O valor de "a" pode ser zero, quando se considera a distribuição Log-Normal a dois parâmetros, ou um valor mínimo da série, quando se considera Log-Normal a três parâmetros. O valor de "b" pode ser o da variável aleatória, quando se considera a probabilidade cumulativa de ocorrência daquele valor (CATALUNHA et al., 2002).

A função densidade de probabilidade da distribuição Gama é definida como (JUNQUEIRA JUNIOR et al., 2007), Equação 4.

$$
F(x)=\frac{1}{\beta^{\alpha} \Gamma(\alpha)} x^{\alpha-1} e^{\frac{x}{\beta}} ; \text { para } 0<x<\infty
$$

em que,

$\mathrm{x}=$ variável aleatória continua;

$\Gamma(\alpha)=$ função Gama do parâmetro alfa;

$\alpha=$ parâmetro de forma da variável aleatória $\mathrm{x}$; e

$\beta=$ parâmetro de escala da variável aleatória $\mathrm{x}$.

Ao ajustar uma distribuição de probabilidade a um conjunto de dados, assume-se a hipótese de que a distribuição pode representar adequadamente o conjunto de informações (CATALUNHA et al., 2002). Para verificar o ajuste entre as frequências de evapotranspiração de referência (ETo mensal observadas e estimadas adotou-se os testes Kolmogorov-Smirnov (KS) e Qui-quadrado $\left(\chi^{2}\right)$, considerando o nível de $5 \%$ de significância.

Por meio das funções de distribuição de probabilidade Normal, Log-Normal e Gama foram geradas os valores máximos esperados de $\mathrm{ET}_{0}$ para os níveis de probabilidade de ocorrência: 50,55 , $60,65,70,75,80,85$ e $90 \%$.

\section{RESULTADOS E DISCUSSÃO}

Por meio da análise de distribuição de frequência foi possível utilizar as funções de distribuição de probabilidade Normal, Log-Normal e Gama para a estimativa de ajuste dos dados de evapotranspiração de referência $\left(E T_{0}\right)$ mensal para o município de Crateús, Ceará. Verifica-se, no Quadro 1, que as distribuições de probabilidade Normal, LogNormal e Gama ajustaram-se aos dados de ET em todos os meses, mediante a aplicação do teste de aderência Kolmogorov-Smirnov (KS) a 5\% de significância, mostrando, assim, que essas distribuições podem ser utilizadas sem que haja nenhuma restrição para épocas especificas do ano.

Segundo Assis et al. (2013), a distribuição Normal deve ser o melhor modelo escolhido pela simplicidade, abrangência, parcimônia e aplicabilidade inferencial, permitindo fazer valiosas inferências, no que se referem às estimativas de valores, previsões probabilísticas, norteando assim todas as atividades que dependem desta variável aleatória climática, particularmente as atividades agropecuárias.

Resultados semelhantes aos observados no presente trabalho foram encontrados por Silva et al. (2013a) e Silva et al. (2014b) nos municípios de Morada Nova e Campos Sales, no estado do Ceará, e por Santos et al. (2014) em Cruz das Almas, Bahia, onde a distribuição Normal ajustou-se aos dados de $\mathrm{ET}_{\mathrm{o}}$ em todos os meses do ano pelo teste KS, em nível de 5\% de significância. Nas condições climáticas de Piracicaba, SP, a distribuição Normal também se mostrou apta estatisticamente para representar os dados de ET (SAAD et al., 2002), assim como para a localidade de Pelotas, RS (BACELAR et al., 2011), mediante a aplicação do teste KS em nível de 5\% de significância.

Já quanto a distribuição Log-Normal, a mesma se mostrou apta para representar a variabilidade da $\mathrm{ET}_{\text {o }}$ mensal, segundo o teste KS em nível de 5\% de significância para a localidade de Iguatu, Ceará (CAVALCANTE JUNIOR et al., 2009).

A distribuição Log-Normal ajustou-se aos dados de $\mathrm{ET}_{\mathrm{o}}$ em todos os meses, mediante a aplicação do teste de aderência Qui-quadrado $\left(\chi^{2}\right)$ a $5 \%$ de significância e as distribuições Normal e Gama ajustaram-se em dez meses, exceto nos meses de fevereiro e junho, respectivamente.

Corroborando o presente trabalho, a distribuição Normal ajustou-se aos dados de ET mensal, a exceção dos meses de abril e agosto nas condições climáticas de Cruz das Almas, BA (SANTOS et al., 2014) e a exceção dos meses de fevereiro e dezembro em Campos Sales, CE (SILVA et al., 2014b), mediante a aplicação do teste $\chi^{2}$ a $5 \%$ de significância. Já na localidade de Morada Nova, no estado do Ceará, houve ajuste em sete meses do ano de acordo com o teste $\chi^{2}$ a 5\% de significância (SILVA et al., 2013a).

No Quadro 2 são apresentados os valores máximos esperados de $\mathrm{ET}_{0}$ mensal obtidos pelas distribuições Normal, Log-Normal e Gama com diferentes níveis de probabilidades de ocorrência para a localidade de Crateús, Ceará. Analisando distribuição Normal em nível de $75 \%$ de probabilidade, geralmente recomendado para o dimensionamento de projetos de irrigação, verifica-se outubro como mês de pico, é esperado um valor máximo de $\mathrm{ET}_{\mathrm{o}}$ da ordem de $6,73 \mathrm{~mm} \mathrm{dia}{ }^{-1}$, ou seja, de cada quatro anos, em três o valor da $\mathrm{ET}_{0}$ será igual ou menor a $6,73 \mathrm{~mm}$ dia 1. Os menores valores de $\mathrm{ET}_{\mathrm{o}}$ concentram-se nos meses de abril e maio, da ordem de 4,05 e 4,08 mm 
Quadro 1. Desempenho das distribuições de probabilidade Normal, Log-Normal e Gama aos dados de ET mensal do município de Crateús, segundo os testes Qui-quadrado $\left(\chi^{2}\right)$ e Kolmogorov-Smirnov (KS) a 5\% de significância

\begin{tabular}{|c|c|c|c|c|c|c|}
\hline \multirow{3}{*}{ Meses } & \multicolumn{2}{|c|}{ Normal } & \multicolumn{2}{|c|}{ Log-Normal } & \multicolumn{2}{|c|}{ Gama } \\
\hline & \multicolumn{6}{|c|}{ Testes de aderência } \\
\hline & $\mathrm{KS}$ & $\chi^{2}$ & KS & $\chi^{2}$ & KS & $\chi^{2}$ \\
\hline Janeiro & AJ & AJ & AJ & AJ & AJ & $\mathrm{AJ}$ \\
\hline Fevereiro & AJ & NA & AJ & AJ & AJ & NA \\
\hline Março & AJ & AJ & AJ & AJ & AJ & $\mathrm{AJ}$ \\
\hline Abril & AJ & AJ & AJ & AJ & AJ & $\mathrm{AJ}$ \\
\hline Maio & AJ & AJ & $\mathrm{AJ}$ & AJ & AJ & $\mathrm{AJ}$ \\
\hline Junho & AJ & NA & AJ & AJ & AJ & NA \\
\hline Julho & AJ & AJ & AJ & AJ & AJ & $\mathrm{AJ}$ \\
\hline Agosto & AJ & AJ & AJ & AJ & AJ & $\mathrm{AJ}$ \\
\hline Setembro & AJ & AJ & $\mathrm{AJ}$ & AJ & AJ & $\mathrm{AJ}$ \\
\hline Outubro & AJ & AJ & AJ & AJ & AJ & $\mathrm{AJ}$ \\
\hline Novembro & AJ & AJ & AJ & AJ & AJ & $\mathrm{AJ}$ \\
\hline Dezembro & AJ & AJ & AJ & AJ & AJ & AJ \\
\hline
\end{tabular}

Em que: AJ - ajustou; NA - não ajustou a respectiva distribuição de probabilidade

dia $^{-1}$, respectivamente.

Na prática, é usual utilizar valor médio mensal da evapotranspiração paradimensionamentode sistemas de irrigação, o que conduz ao subdimensionamento dos mesmos, enquanto a adoção do máximo valor diário da evapotranspiração no período de pico acarreta o superdimensionamento (SAAD et al., 2002), sendo as tabelas geradas no presente trabalho com diferentes níveis de probabilidades um subsídio para consulta por técnicos da região, tanto para o dimensionamento de sistemas de irrigação, bem como para o manejo da irrigação.

Com a utilização da distribuição Log-Normal em nível de $75 \%$ de probabilidade, em outubro, mês de pico, o valor esperado de ET $T_{0}$ é aproximadamente igual ao obtido pela distribuição Normal, de $6,72 \mathrm{~mm} \mathrm{dia}^{-1}$ e os menores valores de ET também concentrando-se nos meses de abril e maio, de 4,05 e 4,07 $\mathrm{mm} \mathrm{dia}^{-}$ ${ }^{1}$, respectivamente. Analisando a distribuição Gama para obtenção dos valores esperados de ET mensal, o valor do mês de pico (outubro) é igual ao obtido pela distribuição Log-Normal, ou seja, de 6,72 $\mathrm{mm} \mathrm{dia}^{-1} \mathrm{e}$ os menores valores de $\mathrm{ET}_{\mathrm{o}}$ também são observados nos meses de abril e maio, de 4,04 e 4,07 $\mathrm{mm} \mathrm{dia}^{-1}$, respectivamente.

No Quadro 3, assim como ocorreu para o município de Crateús, em Quixeramobim no estado do Ceará, ocorreu ajuste das distribuições de probabilidade Normal, Log-Normal e Gama aos dados de $\mathrm{ET}_{\mathrm{o}}$ em todos os meses do ano, pelo teste de aderência KS a 5\% de significância. Resultados similares foram reportados em outros trabalhos, como por Cavalcante Junior et al. (2009) e Silva et al. (2014b) para as localidades de Iguatu e Campos Sales, no estado do Ceará, em que a distribuição Gama ajustou-se aos dados de ET em onze meses do ano, segundo o teste KS em nível de $5 \%$ de significância. Mediante a comprovação pelo teste KS em nível de 5\% de significância, a distribuição Normal mostrou-se apta para representar aos dados de $\mathrm{ET}_{\mathrm{o}}$ mensal para condições climáticas de Mossoró, RN (PEIXOTO et al., 2011a), assim como, para as condições climáticas de Ouricuri, Pernambuco (VELLAME et al., 2012), em que a exceção do mês de maio em todos os demais meses, os dados de $\mathrm{ET}_{\mathrm{o}}$ seguiram a distribuição Normal. Ainda nas condições climáticas de Mossoró, $\mathrm{RN}$, verificou-se que a distribuição Normal foi o 
Quadro 2. Valores prováveis de ocorrência de ET onensal, obtidos pelas distribuições Normal, Log-Normal e Gama, para diferentes níveis de probabilidade em Crateús, Ceará

\begin{tabular}{|c|c|c|c|c|c|c|c|c|c|}
\hline \multirow{2}{*}{ Meses } & \multicolumn{9}{|c|}{ Níveis de Probabilidades } \\
\hline & $50 \%$ & $55 \%$ & $60 \%$ & $65 \%$ & $70 \%$ & $75 \%$ & $80 \%$ & $85 \%$ & $90 \%$ \\
\hline \multicolumn{10}{|c|}{ Normal } \\
\hline Janeiro & 4,91 & 4,98 & 5,05 & 5,12 & 5,20 & 5,28 & 5,37 & 5,48 & 5,62 \\
\hline Fevereiro & 4,66 & 4,75 & 4,83 & 4,91 & 5,00 & 5,10 & 5,21 & 5,33 & 5,49 \\
\hline Março & 4,04 & 4,09 & 4,14 & 4,20 & 4,25 & 4,31 & 4,38 & 4,46 & 4,56 \\
\hline Abril & 3,72 & 3,78 & 3,84 & 3,91 & 3,98 & 4,05 & 4,13 & 4,22 & 4,34 \\
\hline Maio & 3,70 & 3,77 & 3,85 & 3,92 & 4,00 & 4,08 & 4,17 & 4,28 & 4,42 \\
\hline Junho & 4,00 & 4,06 & 4,12 & 4,19 & 4,25 & 4,33 & 4,41 & 4,50 & 4,62 \\
\hline Julho & 4,59 & 4,65 & 4,71 & 4,78 & 4,85 & 4,93 & 5,01 & 5,11 & 5,23 \\
\hline Agosto & 5,53 & 5,59 & 5,65 & 5,71 & 5,77 & 5,84 & 5,92 & 6,01 & 6,13 \\
\hline Setembro & 6,23 & 6,29 & 6,36 & 6,42 & 6,49 & 6,57 & 6,65 & 6,75 & 6,87 \\
\hline Outubro & 6,37 & 6,44 & 6,50 & 6,58 & 6,65 & 6,73 & 6,82 & 6,93 & 7,06 \\
\hline Novembro & 6,20 & 6,27 & 6,35 & 6,43 & 6,52 & 6,61 & 6,72 & 6,84 & 6,99 \\
\hline Dezembro & 5,82 & 5,91 & 6,01 & 6,11 & 6,22 & 6,33 & 6,46 & 6,61 & 6,80 \\
\hline \multicolumn{10}{|c|}{ Log-Normal } \\
\hline Janeiro & 4,88 & 4,95 & 5,02 & 5,10 & 5,18 & 5,26 & 5,36 & 5,48 & 5,64 \\
\hline Fevereiro & 4,62 & 4,70 & 4,78 & 4,87 & 4,96 & 5,06 & 5,17 & 5,31 & 5,49 \\
\hline Março & 4,02 & 4,07 & 4,12 & 4,17 & 4,23 & 4,29 & 4,36 & 4,45 & 4,55 \\
\hline Abril & 3,69 & 3,76 & 3,82 & 3,89 & 3,97 & 4,05 & 4,15 & 4,26 & 4,41 \\
\hline Maio & 3,66 & 3,73 & 3,81 & 3,89 & 3,98 & 4,07 & 4,18 & 4,31 & 4,48 \\
\hline Junho & 3,97 & 4,03 & 4,09 & 4,16 & 4,22 & 4,30 & 4,38 & 4,49 & 4,62 \\
\hline Julho & 4,56 & 4,62 & 4,69 & 4,75 & 4,83 & 4,90 & 4,99 & 5,10 & 5,24 \\
\hline Agosto & 5,51 & 5,57 & 5,63 & 5,69 & 5,76 & 5,84 & 5,92 & 6,02 & 6,15 \\
\hline Setembro & 6,21 & 6,28 & 6,34 & 6,41 & 6,48 & 6,56 & 6,65 & 6,75 & 6,89 \\
\hline Outubro & 6,35 & 6,41 & 6,48 & 6,56 & 6,63 & 6,72 & 6,82 & 6,93 & 7,08 \\
\hline Novembro & 6,17 & 6,25 & 6,33 & 6,42 & 6,51 & 6,61 & 6,73 & 6,86 & 7,04 \\
\hline Dezembro & 5,77 & 5,86 & 5,96 & 6,06 & 6,17 & 6,29 & 6,42 & 6,58 & 6,79 \\
\hline \multicolumn{10}{|c|}{ Gama } \\
\hline Janeiro & 4,89 & 4,96 & 5,03 & 5,10 & 5,18 & 5,26 & 5,36 & 5,47 & 5,62 \\
\hline Fevereiro & 4,64 & 4,72 & 4,80 & 4,88 & 4,97 & 5,07 & 5,18 & 5,31 & 5,48 \\
\hline Março & 4,03 & 4,08 & 4,13 & 4,18 & 4,24 & 4,30 & 4,36 & 4,44 & 4,55 \\
\hline Abril & 3,70 & 3,76 & 3,83 & 3,89 & 3,97 & 4,04 & 4,13 & 4,24 & 4,37 \\
\hline Maio & 3,67 & 3,75 & 3,82 & 3,90 & 3,98 & 4,07 & 4,17 & 4,29 & 4,44 \\
\hline Junho & 3,98 & 4,04 & 4,10 & 4,16 & 4,23 & 4,30 & 4,39 & 4,48 & 4,61 \\
\hline Julho & 4,57 & 4,63 & 4,69 & 4,76 & 4,83 & 4,91 & 4,99 & 5,09 & 5,22 \\
\hline Agosto & 5,51 & 5,57 & 5,63 & 5,69 & 5,76 & 5,83 & 5,92 & 6,01 & 6,13 \\
\hline Setembro & 6,22 & 6,28 & 6,34 & 6,41 & 6,48 & 6,56 & 6,64 & 6,74 & 6,87 \\
\hline Outubro & 6,35 & 6,42 & 6,49 & 6,56 & 6,64 & 6,72 & 6,81 & 6,92 & 7,06 \\
\hline Novembro & 6,18 & 6,25 & 6,34 & 6,42 & 6,51 & 6,61 & 6,71 & 6,84 & 7,01 \\
\hline Dezembro & 5,78 & 5,88 & 5,97 & 6,07 & 6,18 & 6,29 & 6,42 & 6,58 & 6,77 \\
\hline
\end{tabular}


melhor modelo para representar o comportamento da evapotranspiração de referência, devido à simplicidade da sua equação, de baixo número de parâmetros a ser estimado, facilitar a estimativa, a sua ampla utilização nos estudos de inferência estatística, assim como a maior percentagem de adesão a série estudada (ASSIS; SOUSA, 2013; ASSIS et al., 2014). No estado de São Paulo, a distribuição Normal apresentou melhor desempenho nos períodos quinzenais avaliados, em comparação às distribuições Log-Normal e Gama (BLAIN; BRUNINI, 2007).

Nos meses de junho, agosto e dezembro a distribuição de probabilidade Normal não se ajustou aos dados de $\mathrm{ET}_{\mathrm{o}}$ mensal pelo o teste $\chi^{2}$ a $5 \%$ de significância. Já as distribuições LogNormal e Gama não se ajustaram nos meses junho, agosto, setembro e dezembro. No estudo de Almeida et al. (2009) no munícipio de Iguatu, CE, a distribuição Gama mostrou apta estatisticamente para representar os dados de $\mathrm{ET}_{\mathrm{o}}$ em escala pentadial mediante a comprovação pelo teste $\chi^{2}$ a $5 \%$ de significância. Em comprovação por este mesmo teste de aderência (5\% de significância), nos estudos de Santos et al. (2014) e Silva et al. (2014b) houve ajuste da distribuição Gama em nove meses do ano.

Os valores esperados de $\mathrm{ET}_{\mathrm{o}}$ mensal obtidos utilizando-se as distribuições Normal, Log-Normal e Gama com diferentes níveis de probabilidades de ocorrência para a localidade de Quixeramobim são apresentados no Quadro 4. Analisando distribuição Normal com nível de $75 \%$ de probabilidade, verifica-se a ET de pico no mês de outubro, sendo esperado valor máxima de $7,71 \mathrm{~mm} \mathrm{dia}^{-1}$, ou seja, de cada quatro anos, em três, o valor da $\mathrm{ET}_{0}$ será igual ou menor a 7,71 $\mathrm{mm} \mathrm{dia}^{-1}$. A ET mínima ocorreu no mês de maio, de 4,08 $\mathrm{mm} \mathrm{dia}^{-1}$. Por meio da utilização da distribuição Log-Normal, a ET máxima esperada é de 7,72 $\mathrm{mm} \mathrm{dia}^{-1}$ e mínima de 4,05 $\mathrm{mm} \mathrm{dia}^{-1}$, concentrando-se no mês de maio. A ET máxima esperada obtida pela distribuição Gama é igual a obtida pela distribuição Normal, de7,71 $\mathrm{mm}_{\text {dia }}{ }^{-1}$ e a mínima de 4,06 $\mathrm{mm} \mathrm{dia}^{-1}$.

Nas condições climáticas de Tauá (Quadro 5), assim como observado nos municípios de Crateús e Quixeramobim no estado do Ceará, as distribuições de probabilidade Normal, Log-Normal e Gama

Quadro 3. Desempenho das distribuições de probabilidade Normal, Log-Normal e Gama aos dados de ET mensal do município de Quixeramobim, segundo os testes Qui-quadrado $\left(\chi^{2}\right)$ e KolmogorovSmirnov (KS) a 5\% de significância

\begin{tabular}{lcccccc}
\hline \multirow{2}{*}{ Meses } & \multicolumn{2}{c}{ Normal } & \multicolumn{2}{c}{ Log-Normal } & \multicolumn{2}{c}{ Gama } \\
\cline { 2 - 7 } & & & \multicolumn{2}{c}{ Testes de aderência } & \\
\cline { 2 - 7 } Janeiro & KS & $\chi^{2}$ & KS & $\chi^{2}$ & KS & $\chi^{2}$ \\
Fevereiro & AJ & AJ & AJ & AJ & AJ & AJ \\
Março & AJ & AJ & AJ & AJ & AJ & AJ \\
Abril & AJ & AJ & AJ & AJ & AJ & AJ \\
Maio & AJ & AJ & AJ & AJ & AJ & AJ \\
Junho & AJ & AJ & AJ & AJ & AJ & AJ \\
Julho & AJ & NA & AJ & NA & AJ & NA \\
Agosto & AJ & AJ & AJ & AJ & AJ & AJ \\
Setembro & AJ & NA & AJ & NA & AJ & NA \\
Outubro & AJ & AJ & AJ & NA & AJ & NA \\
Novembro & AJ & AJ & AJ & AJ & AJ & AJ \\
Dezembro & AJ & AJ & AJ & AJ & AJ & AJ \\
\hline
\end{tabular}

Em que: AJ - ajustou; NA - não ajustou a respectiva distribuição de probabilidade 
Quadro 4. Valores prováveis de ocorrência de ET mensal, obtidos pelas distribuições Normal, Log-Normal e Gama, para diferentes níveis de probabilidade em Quixeramobim, Ceará

\begin{tabular}{|c|c|c|c|c|c|c|c|c|c|}
\hline \multirow{2}{*}{ Meses } & \multicolumn{9}{|c|}{ Níveis de Probabilidades } \\
\hline & $50 \%$ & $55 \%$ & $60 \%$ & $65 \%$ & $70 \%$ & $75 \%$ & $80 \%$ & $85 \%$ & $90 \%$ \\
\hline \multicolumn{10}{|c|}{ Normal } \\
\hline Janeiro & 5,77 & 5,88 & 6,00 & 6,12 & 6,24 & 6,37 & 6,52 & 6,69 & 6,91 \\
\hline Fevereiro & 5,18 & 5,27 & 5,37 & 5,46 & 5,57 & 5,68 & 5,80 & 5,95 & 6,13 \\
\hline Março & 4,54 & 4,61 & 4,68 & 4,75 & 4,82 & 4,91 & 4,99 & 5,10 & 5,23 \\
\hline Abril & 4,07 & 4,13 & 4,19 & 4,25 & 4,32 & 4,39 & 4,47 & 4,57 & 4,69 \\
\hline Maio & 3,79 & 3,84 & 3,90 & 3,95 & 4,01 & 4,08 & 4,15 & 4,23 & 4,34 \\
\hline Junho & 3,90 & 3,99 & 4,08 & 4,17 & 4,26 & 4,36 & 4,48 & 4,61 & 4,78 \\
\hline Julho & 4,33 & 4,41 & 4,49 & 4,58 & 4,68 & 4,77 & 4,89 & 5,02 & 5,18 \\
\hline Agosto & 5,56 & 5,66 & 5,75 & 5,85 & 5,95 & 6,06 & 6,18 & 6,33 & 6,51 \\
\hline Setembro & 6,67 & 6,77 & 6,88 & 6,99 & 7,10 & 7,22 & 7,36 & 7,52 & 7,72 \\
\hline Outubro & 7,14 & 7,24 & 7,35 & 7,46 & 7,58 & 7,71 & 7,85 & 8,02 & 8,22 \\
\hline Novembro & 7,00 & 7,08 & 7,16 & 7,24 & 7,33 & 7,43 & 7,54 & 7,66 & 7,82 \\
\hline Dezembro & 6,51 & 6,60 & 6,69 & 6,79 & 6,89 & 7,00 & 7,12 & 7,27 & 7,45 \\
\hline \multicolumn{10}{|c|}{ Log-Normal } \\
\hline Janeiro & 5,71 & 5,82 & 5,94 & 6,06 & 6,19 & 6,34 & 6,51 & 6,71 & 6,97 \\
\hline Fevereiro & 5,13 & 5,22 & 5,32 & 5,42 & 5,53 & 5,65 & 5,78 & 5,95 & 6,16 \\
\hline Março & 4,52 & 4,58 & 4,64 & 4,71 & 4,78 & 4,86 & 4,94 & 5,05 & 5,18 \\
\hline Abril & 4,04 & 4,10 & 4,16 & 4,23 & 4,29 & 4,37 & 4,46 & 4,56 & 4,69 \\
\hline Maio & 3,77 & 3,82 & 3,87 & 3,93 & 3,99 & 4,05 & 4,13 & 4,21 & 4,33 \\
\hline Junho & 3,85 & 3,93 & 4,01 & 4,10 & 4,19 & 4,29 & 4,40 & 4,54 & 4,73 \\
\hline Julho & 4,28 & 4,36 & 4,44 & 4,53 & 4,62 & 4,72 & 4,84 & 4,98 & 5,16 \\
\hline Agosto & 5,51 & 5,61 & 5,71 & 5,82 & 5,93 & 6,05 & 6,20 & 6,37 & 6,59 \\
\hline Setembro & 6,61 & 6,73 & 6,85 & 6,97 & 7,10 & 7,25 & 7,42 & 7,62 & 7,87 \\
\hline Outubro & 7,08 & 7,20 & 7,32 & 7,44 & 7,57 & 7,72 & 7,89 & 8,09 & 8,35 \\
\hline Novembro & 6,97 & 7,05 & 7,13 & 7,22 & 7,32 & 7,42 & 7,53 & 7,67 & 7,85 \\
\hline Dezembro & 6,47 & 6,56 & 6,66 & 6,76 & 6,88 & 7,00 & 7,14 & 7,30 & 7,52 \\
\hline \multicolumn{10}{|c|}{ Gama } \\
\hline Janeiro & 5,73 & 5,84 & 5,95 & 6,07 & 6,20 & 6,34 & 6,50 & 6,69 & 6,93 \\
\hline Fevereiro & 5,14 & 5,24 & 5,33 & 5,43 & 5,53 & 5,65 & 5,78 & 5,93 & 6,13 \\
\hline Março & 4,53 & 4,59 & 4,65 & 4,72 & 4,79 & 4,87 & 4,95 & 5,06 & 5,19 \\
\hline Abril & 4,05 & 4,11 & 4,17 & 4,23 & 4,30 & 4,37 & 4,46 & 4,55 & 4,68 \\
\hline Maio & 3,78 & 3,83 & 3,88 & 3,93 & 3,99 & 4,06 & 4,13 & 4,21 & 4,32 \\
\hline Junho & 3,87 & 3,95 & 4,03 & 4,12 & 4,21 & 4,31 & 4,42 & 4,56 & 4,73 \\
\hline Julho & 4,30 & 4,38 & 4,46 & 4,54 & 4,63 & 4,73 & 4,85 & 4,98 & 5,15 \\
\hline Agosto & 5,53 & 5,62 & 5,72 & 5,82 & 5,93 & 6,05 & 6,18 & 6,34 & 6,54 \\
\hline Setembro & 6,63 & 6,74 & 6,85 & 6,97 & 7,09 & 7,23 & 7,38 & 7,57 & 7,80 \\
\hline Outubro & 7,10 & 7,21 & 7,32 & 7,44 & 7,57 & 7,71 & 7,86 & 8,05 & 8,28 \\
\hline Novembro & 6,98 & 7,06 & 7,14 & 7,23 & 7,32 & 7,42 & 7,53 & 7,66 & 7,83 \\
\hline Dezembro & 6,48 & 6,57 & 6,67 & 6,77 & 6,88 & 6,99 & 7,12 & 7,28 & 7,48 \\
\hline
\end{tabular}


ajustaram-se aos dados de ET mensal para todos os meses do ano, segundo o teste KS a $5 \%$ de significância. Aproximadamente $65 \%$ dos valores decendiais de $\mathrm{ET}_{0}$ ajustaram-se a distribuição Normal pelo teste KS a 5\% de significância, nas condições climáticas da região de Telêmaco Borba, PR (JERSZURKI et al., 2012). Já na localidade de Sete Lagoas, MG, a distribuição Normal ajustou-se aos dados de evapotranspiração de referência em quatro meses, pelo teste KS a 5\% de significância (BORGES JÚNIOR et al., 2013). Os autores ainda ressaltam a facilidade para obtenção de parâmetros desta distribuição, bem como para sua aplicação.

As distribuições de probabilidade Gama e LogNormal proporcionaram ajuste altamente satisfatório aos dados de evapotranspiração potencial mensal no estado do Paraná (ABUMANSSUR, 2006). Para condições climáticas de Mossoró, RN, o modelo de distribuição Log-Normal pode ser usado para estimar a probabilidade de ocorrência de ET média para períodos mensais, segundo o teste KS (SANTOS et al., 2011; PEIXOTO et al., 2011b). Nas condições climáticas de Petrolina, PE, a distribuição Gama mostrou-se apta estatisticamente para representar os dados de ET em escala quinzenal mediante a comprovação pelo teste KS a 5\% (SILVA et al., 2014a) e em escala pentadial a 20\% de significância (SOUZA et al., 2014).

A exceção do mês de agosto, as distribuições de probabilidade Normal, Log-Normal e Gama se ajustaram aos dados de ET mensal, mediante a aplicação do teste $\chi^{2}$ a $5 \%$ de significância. Mediante a aplicação do mesmo teste de aderência e nível de significância, Silva et al. (2014b) reportaram ajuste com a distribuição Log-Normal em nove meses do ano, nas condições climáticas de Campos Sales, Ceará.

Analisando os resultados obtidos utilizandose a distribuição Normal com nível de $75 \%$ de probabilidade para a localidade de Tauá, em outubro o mês de pico, é esperado um valor máximo de ET de $6,27 \mathrm{~mm} \mathrm{dia}^{-1}$, ou seja, de cada quatro anos, em três, o valor da ET será igual ou menor a $6,27 \mathrm{~mm}$ dia $^{-1}$. A ET mínima concentra-se no mês de maio, de 4,0 $\mathrm{mm} \mathrm{dia}^{-1}$ (Quadro 6). Com a utilização da distribuição Log-Normal, a ET máxima esperada é de $6,30 \mathrm{~mm}$ dia $^{-1}$ e mínima de $3,98 \mathrm{~mm} \mathrm{dia}{ }^{-1}$, concentrando-se no mês de maio.

Quadro 5. Desempenho das distribuições de probabilidade Normal, Log-Normal e Gama aos dados de ET mensal do município de Tauá, segundo os testes Qui-quadrado $\left(\chi^{2}\right)$ e Kolmogorov-Smirnov (KS) a 5\% de significância

\begin{tabular}{|c|c|c|c|c|c|c|}
\hline \multirow{3}{*}{ Meses } & \multicolumn{2}{|c|}{ Normal } & \multicolumn{2}{|c|}{ Log-Normal } & \multicolumn{2}{|c|}{ Gama } \\
\hline & \multicolumn{6}{|c|}{ Testes de aderência } \\
\hline & KS & $\chi^{2}$ & KS & $\chi^{2}$ & KS & $\chi^{2}$ \\
\hline Janeiro & AJ & AJ & AJ & AJ & AJ & $\mathrm{AJ}$ \\
\hline Fevereiro & AJ & AJ & AJ & AJ & AJ & AJ \\
\hline Março & AJ & AJ & AJ & AJ & AJ & $\mathrm{AJ}$ \\
\hline Abril & AJ & AJ & AJ & AJ & AJ & $\mathrm{AJ}$ \\
\hline Maio & $\mathrm{AJ}$ & $\mathrm{AJ}$ & AJ & AJ & AJ & AJ \\
\hline Junho & AJ & AJ & AJ & AJ & AJ & $\mathrm{AJ}$ \\
\hline Julho & AJ & AJ & AJ & AJ & AJ & $\mathrm{AJ}$ \\
\hline Agosto & AJ & NA & AJ & NA & AJ & NA \\
\hline Setembro & AJ & AJ & AJ & AJ & AJ & AJ \\
\hline Outubro & AJ & AJ & AJ & AJ & AJ & $\mathrm{AJ}$ \\
\hline Novembro & $\mathrm{AJ}$ & AJ & $\mathrm{AJ}$ & AJ & AJ & AJ \\
\hline Dezembro & AJ & AJ & AJ & AJ & AJ & AJ \\
\hline
\end{tabular}

Em que: AJ - ajustou; NA - não ajustou a respectiva distribuição de probabilidade 
PROBABILIDADE DE OCORRÊNCIA DE VALORES ESTIMADOS DE EVAPOTRANSPIRAÇÃO DE REFERÊNCIA ...

Quadro 6. Valores prováveis de ocorrência de ET mensal, obtidos pelas distribuições Normal, Log-Normal e Gama, para diferentes níveis de probabilidade em Tauá, Ceará

\begin{tabular}{|c|c|c|c|c|c|c|c|c|c|}
\hline \multirow{2}{*}{ Meses } & \multicolumn{9}{|c|}{ Níveis de Probabilidades } \\
\hline & $50 \%$ & $55 \%$ & $60 \%$ & $65 \%$ & $70 \%$ & $75 \%$ & $80 \%$ & $85 \%$ & $90 \%$ \\
\hline \multicolumn{10}{|c|}{ Normal } \\
\hline Janeiro & 4,65 & 4,74 & 4,82 & 4,91 & 5,01 & 5,11 & 5,22 & 5,35 & 5,52 \\
\hline Fevereiro & 4,32 & 4,39 & 4,47 & 4,55 & 4,63 & 4,72 & 4,82 & 4,94 & 5,09 \\
\hline Março & 4,06 & 4,12 & 4,18 & 4,25 & 4,31 & 4,39 & 4,46 & 4,56 & 4,67 \\
\hline Abril & 3,73 & 3,79 & 3,85 & 3,91 & 3,97 & 4,04 & 4,12 & 4,21 & 4,33 \\
\hline Maio & 3,70 & 3,76 & 3,81 & 3,87 & 3,93 & 4,00 & 4,07 & 4,16 & 4,27 \\
\hline Junho & 3,87 & 3,93 & 3,99 & 4,06 & 4,12 & 4,20 & 4,28 & 4,37 & 4,49 \\
\hline Julho & 4,26 & 4,33 & 4,40 & 4,48 & 4,56 & 4,64 & 4,74 & 4,85 & 4,98 \\
\hline Agosto & 5,07 & 5,16 & 5,25 & 5,34 & 5,44 & 5,55 & 5,67 & 5,81 & 5,99 \\
\hline Setembro & 5,58 & 5,66 & 5,74 & 5,83 & 5,92 & 6,02 & 6,13 & 6,25 & 6,41 \\
\hline Outubro & 5,66 & 5,77 & 5,89 & 6,01 & 6,13 & 6,27 & 6,42 & 6,59 & 6,81 \\
\hline Novembro & 5,53 & 5,63 & 5,73 & 5,83 & 5,93 & 6,05 & 6,18 & 6,33 & 6,52 \\
\hline Dezembro & 5,19 & 5,29 & 5,38 & 5,48 & 5,59 & 5,70 & 5,83 & 5,97 & 6,16 \\
\hline \multicolumn{10}{|c|}{ Log-Normal } \\
\hline Janeiro & 4,60 & 4,69 & 4,78 & 4,88 & 4,99 & 5,11 & 5,24 & 5,41 & 5,62 \\
\hline Fevereiro & 4,28 & 4,35 & 4,43 & 4,52 & 4,61 & 4,71 & 4,82 & 4,95 & 5,13 \\
\hline Março & 4,04 & 4,10 & 4,16 & 4,23 & 4,30 & 4,37 & 4,46 & 4,56 & 4,70 \\
\hline Abril & 3,70 & 3,76 & 3,82 & 3,88 & 3,95 & 4,03 & 4,12 & 4,22 & 4,35 \\
\hline Maio & 3,68 & 3,73 & 3,79 & 3,84 & 3,91 & 3,98 & 4,05 & 4,15 & 4,27 \\
\hline Junho & 3,84 & 3,90 & 3,96 & 4,03 & 4,10 & 4,17 & 4,26 & 4,37 & 4,50 \\
\hline Julho & 4,22 & 4,30 & 4,37 & 4,45 & 4,54 & 4,63 & 4,74 & 4,87 & 5,03 \\
\hline Agosto & 5,01 & 5,11 & 5,21 & 5,31 & 5,43 & 5,55 & 5,70 & 5,87 & 6,09 \\
\hline Setembro & 5,54 & 5,62 & 5,71 & 5,80 & 5,90 & 6,01 & 6,14 & 6,28 & 6,47 \\
\hline Outubro & 5,58 & 5,71 & 5,84 & 5,98 & 6,14 & 6,30 & 6,50 & 6,73 & 7,03 \\
\hline Novembro & 5,48 & 5,58 & 5,68 & 5,79 & 5,91 & 6,04 & 6,19 & 6,37 & 6,60 \\
\hline Dezembro & 5,14 & 5,24 & 5,34 & 5,44 & 5,56 & 5,68 & 5,83 & 6,00 & 6,23 \\
\hline \multicolumn{10}{|c|}{ Gama } \\
\hline Janeiro & 4,62 & 4,70 & 4,79 & 4,89 & 4,99 & 5,10 & 5,22 & 5,37 & 5,56 \\
\hline Fevereiro & 4,29 & 4,37 & 4,44 & 4,52 & 4,61 & 4,70 & 4,81 & 4,94 & 5,10 \\
\hline Março & 4,05 & 4,11 & 4,17 & 4,23 & 4,30 & 4,37 & 4,46 & 4,55 & 4,68 \\
\hline Abril & 3,71 & 3,77 & 3,83 & 3,89 & 3,96 & 4,03 & 4,11 & 4,21 & 4,33 \\
\hline Maio & 3,68 & 3,74 & 3,79 & 3,85 & 3,91 & 3,98 & 4,05 & 4,14 & 4,26 \\
\hline Junho & 3,85 & 3,91 & 3,97 & 4,03 & 4,10 & 4,18 & 4,26 & 4,36 & 4,49 \\
\hline Julho & 4,24 & 4,31 & 4,38 & 4,46 & 4,54 & 4,63 & 4,73 & 4,85 & 5,00 \\
\hline Agosto & 5,03 & 5,12 & 5,22 & 5,32 & 5,43 & 5,54 & 5,67 & 5,83 & 6,03 \\
\hline Setembro & 5,55 & 5,63 & 5,72 & 5,81 & 5,90 & 6,01 & 6,12 & 6,26 & 6,44 \\
\hline Outubro & 5,61 & 5,73 & 5,85 & 5,98 & 6,12 & 6,27 & 6,45 & 6,65 & 6,92 \\
\hline Novembro & 5,49 & 5,59 & 5,69 & 5,80 & 5,91 & 6,03 & 6,17 & 6,34 & 6,55 \\
\hline Dezembro & 5,16 & 5,25 & 5,35 & 5,45 & 5,56 & 5,68 & 5,82 & 5,98 & 6,18 \\
\hline
\end{tabular}




\section{CONCLUSÕES}

- Nas localidades de Crateús, Quixeramobim e Tauá as distribuições Normal, Log-Normal e Gama se ajustaram aos dados mensais de evapotranspiração de referência (ETo em todos os meses do ano, segundo o teste de aderência Kolmogorov-Smirnov (KS) a 5\% de significância;

- Em Crateús, a distribuição Log-Normal se ajustou em todos os meses aos dados mensais de $\mathrm{ET}_{\mathrm{o}}$ e as distribuições Normal e Gama não se ajustaram apenas nos meses de fevereiro e junho, segundo o teste Qui-quadrado $\left(\chi^{2}\right)$;

- Em Quixeramobim, a distribuição Normal não obteve êxito no ajuste aos dados mensais de $\mathrm{ET}_{\mathrm{o}}$ dos meses de junho, agosto e dezembro e as distribuições Log-Normal e Gama nos meses de junho, agosto, setembro e dezembro, pelo teste de aderência $\chi^{2}$;

- Em Tauá, a exceção do mês de agosto, as distribuições Normal, Log-Normal e Gama ajustaram-se aos dados mensais de $\mathrm{ET}_{\mathrm{o}}$ nos demais meses, segundo o teste $\chi^{2}$;

- Os valores prováveis de probabilidade de ocorrência da $\mathrm{ET}_{\mathrm{o}}$ mensal obtidos no trabalho poderão ser utilizados como parâmetro no dimensionamento de sistemas de irrigação na região dos Sertões no estado do Ceará.

\section{REFERÊNCIAS BIBLIOGRÁFICAS}

ABUMANSSUR, C. Estimativa da evapotranspiração mensal no estado do Paraná. 2006. 103f. Dissertação (Mestrado em Engenharia Agrícola) - Universidade do Oeste do Paraná, Cascavel, 2006.

ALLEN, R.G.; PEREIRA, L.; RAES, D.; SMITH, M. Crop evapotranspiration: guidelines for computing crop water requirements. Rome: FAO, 1998. 300p. (Irrigation and Drainage Paper, 56).

ALMEIDA, B.M.; OLIVEIRA, J.B.; SILVA, I.N.; ARAÚJO, E.M.; OLIVEIRA, A.D. Estudo da distribuição de frequência da evapotranspiração de referência pentadial para a cidade de Iguatu-CE. In: Congresso Brasileiro de Agrometeorologia, 16, 2009. Belo Horizonte. Anais... Belo Horizonte, 2009. 5 p.

ASSIS, J.P.; SOUSA, R.P. Modelagem probabilística para a evapotranspiração potencial mensal, em Mossoró - RN. In: Congresso Brasileiro de Agrometeorologia, 18, 2013. Belém. Anais... Belém, 2013. 5p.

ASSIS, J.P.; SOUSA, R.P.; BEZERRA NETO, F.; LINHARES, P.C.F. Tables of probabilities of reference evapotranspiration for the region of Mossoró, RN, Brazil. Revista Verde de Agroecologia e Desenvolvimento Sustentável, Pombal, v.9, n.3, p.58-67, 2014.

ASSIS, J.P.; SOUSA, R.P.; SILVA, R.M.; LINHARES, P.C.F. Ajuste de sete modelos de distribuições densidade de probabilidade às séries históricas de umidade relativa mensal em Mossoró - RN. Revista Verde de Agroecologia e Desenvolvimento Sustentável, Mossoró, v.8, n.1, p.1-10, 2013.

BACELAR, L.C.S.D.; TEIXEIRA, C.F.A.; DAMÉ, R.C.F.; CORSEUIL, C.W.; KOEFENDER, J. Estimativa da evapotranspiração de referência máxima possível mediante modelo probabilístico para a localidade de Pelotas/RS. In: Congresso Brasileiro de Agrometeorologia, 17, 2011. Guarapari. Anais... Guarapari, 2011. 5p.

BACK, A.J. Variação da evapotranspiração de referência calculada em diferentes intervalos de tempo. Engenharia Agrícola, Jaboticabal, v.27, n.1, p.139-145, 2007.

BLAIN, G.C; BRUNINI, O. Caracterização do regime de evapotranspiração real, em escala decendial, no estado de São Paulo. Revista Brasileira de Meteorologia, Rio de Janeiro, v.22, n.1, p.75-82, 2007.

CATALUNHA, M.J.; SEDIYAMA, G.C.; LEAL, B.G.; SOARES, C.P.B.; RIBEIRO, A. Aplicação 
de cinco funções densidade de probabilidade a séries de precipitação pluvial no estado de Minas Gerais. Revista Brasileira de Agrometeorologia, Santa Maria, v.10, n.1, p.153-162, 2002.

BORGES JÚNIOR, J.C.F.; STEIDLE NETO, A.J.; ANDRADE, C.L.T.; NASCIMENTO, P.T.; BOGGIONE, I.M. Análise de aderência de distribuições de probabilidade a dados de evapotranspiração de referência em Sete Lagoas, MG. In: Congresso Brasileiro de Engenharia Agrícola, 42, 2013, Fortaleza. Anais... Fortaleza, 2013. 4p.

CAVALCANTE JUNIOR, E.G.; OLIVEIRA, J.B.; ALMEIDA, B.M.; ARAÚJO, E.M.; SILVA, I.N. Probabilidade de ocorrência da evapotranspiração de referência, em escala mensal, na região de Iguatu, CE. In: Congresso Brasileiro de Agrometeorologia, 16, 2009. Belo Horizonte. Anais... Belo Horizonte, 2009. 5p.

JERSZURKI, D.; SOUZA, J.L.M.; EVANGELISTA, A.W.P. Evapotranspiração de referência: Distribuição de probabilidade e decomposição de séries temporais para a região de Telêmaco Borba, Estado do Paraná. In: Reunião Sul-Brasileira de Ciência do Solo, 9, 2012, Lages. Anais... Lages, 2012. 4p.

JUNQUEIRA JÚNIOR, J.A.; GOMES, N.M.; MELLO, C.R.; SILVA, A.M. Precipitação provável para a região de Madre de Deus, Alto Rio Grande: modelos de probabilidades e valores característicos. Ciência e Agrotecnologia, Lavras, v.31, n.3, p.842-850, 2007.

LYRA, G.B.; GARCIA, B.I.L.; PIEDADE, S.M.S.; SEDIYAMA, G.C.; SENTELHAS, P.C. Regiões homogêneas e funções de distribuição de probabilidade da precipitação pluvial no Estado de Táchira, Venezuela. Pesquisa Agropecuária Brasileira, Brasília, v.41, n.2, p.205-215, 2006.

PEIXOTO, T.D.C.; LEVIEN, S.L.A.; ANDRE, H.F.B. Probabilidade de ocorrência da evapotranspiração de referência média em Mossoró-RN, utilizando o modelo de distribuição probabilístico Normal. In: II Reunião para Manejo e Sustentabilidade da Irrigação em Regiões Áridas e Semiáridas, 2011, Cruz das Almas - BA. Anais... Cruz das Almas - BA, 2011a. p.85-88.

PEIXOTO, T.D.C.; LEVIEN, S.L.A.; ANDRE, H.F.B. Probabilidade de ocorrência da evapotranspiração de referência média em Mossoró-RN, utilizando o modelo de distribuição probabilístico Log Normal. In: II Reunião para Manejo e Sustentabilidade da Irrigação em Regiões Áridas e Semiáridas, 2011, Cruz das Almas - BA. Anais... Cruz das Almas - BA, 2011b. p.89-92.

PRELA-PANTANO, A.; ROLIM, G.S.; CAMARGO, M.B.P. Probabilidade de ocorrência de temperaturas mínimas menores que $5^{\circ} \mathrm{C}$ na região do médio Paranapanema. Bragantia, Campinas, v.68, n.1, p.279-284, 2009.

RIBEIRO, B.T.; AVANZI, J.C.; MELLO, C.R.; LIMA, J.M.; SILVA, M.L.N. Comparação de distribuições de probabilidade e estimativa da precipitação provável para região de Barbacena, MG. Ciência e Agrotecnologia, Lavras, v.31, n.5, p.1297-1302, 2007.

SAAD, J.C.C.; BISCARO, G.A.; DELMANTO JUNIOR, O.; FRIZZONE, J.A. Estudo da distribuição da evapotranspiração de referência visando o dimensionamento de sistemas de irrigação. Irriga, Botucatu, v.7, n.1, p.10-17, 2002.

SANTOS, L.C.; ZOCOLER, J.L.; BEZERRA, A.V.; JUSTI, A.L.; CARVALHO, J.J. Estudo da demanda evapotranspirométrica do munícipio de Vitória da Conquista/BA. Revista Brasileira de Agricultura Irrigada, Fortaleza, v.5, n.3, p.173187, 2011.

SANTOS, N.T.; SILVA, M.G.; OLIVEIRA, A.S.; SILVA FILHO, J.A.; PEREIRA, F.A.C.; BATISTA, L.S. Distribuições de probabilidade da evapotranspiração de referência em Cruz das Almas, Bahia. In: II Brazilian Symposium on Salinity \& II Brazilian Meeting on Irrigation Engineering, 2014, Fortaleza. Anais... II INOVAGRI International Meeting: Fortaleza, 2014. p.4407-4413. 
SANTOS, W.O.; BATISTA, B.D.O.; ESPINOLA SOBRINHO, J.; PEREIRA, V.C.; CAVALCANTE JUNIOR, E.G.; ALMEIDA, B.M. Ajuste de modelos de distribuições de probabilidade a série histórica de 1970 a 2007 de evapotranspiração de referência (ETo) em Mossoró-RN. . In: II Reunião para Manejo e Sustentabilidade da Irrigação em Regiões Áridas e Semiáridas, 2011, Cruz das Almas - BA. Anais... Cruz das Almas - BA, 2011. p.185-188.

SILVA, F.C.; FIETZ, C.R.; FOLEGATTI, M.V.; PEREIRA, F.A.C. Distribuição e frequência da evapotranspiração de referência de Cruz das Almas, BA. Revista Brasileira de Engenharia Agrícola e Ambiental, Campina Grande, v.2, n.3, p.284-286, 1998.

SILVA, A.O.; CORREIA, J.S.; BASSOI, L.H.; TEIXEIRA, A.H.C. Evapotranspiração de referência para dimensionamento de sistemas de irrigação em Petrolina, PE. In: II Brazilian Symposium on Salinity \& II Brazilian Meeting on Irrigation Engineering, 2014, Fortaleza. Anais... II INOVAGRI International Meeting: Fortaleza, 2014a. p.2076-2082.

SILVA, M.G.; ARRAES, F.D.D.; LEDO, E.R.F.; SANTOS, N.T.; SILVA FILHO, J.A. Avaliação da evapotranspiração de referência por PenmanMonteith usando dados climáticos mínimos no sertão do Ceará. Revista Agro@mbiente On-line,
Boa Vista, v.7, n.3, p.284-293, 2013b.

SILVA, M.G.; CARMO, F.F.; LÊDO, E.R.F.; SILVA FILHO, J.A.; SANTOS, K.S.; SANTOS, N.T. Probabilidade de ocorrência da evapotranspiração de referência mensal na região de Campos Sales, Ceará. In: II Brazilian Symposium on Salinity \& II Brazilian Meeting on Irrigation Engineering, 2014, Fortaleza. Anais... II INOVAGRI International Meeting: Fortaleza, 2014b. p.341-350.

SILVA, M.G.; LEDO, E.R.F.; SANTOS, N.T.; BATISTA, L.S.; SILVA FILHO, J.A. Distribuição de probabilidade da evapotranspiração de referência mensal em Morada Nova-CE. In: Congresso Brasileiro de Engenharia Agrícola, 42, 2013, Fortaleza. Anais... Fortaleza, 2013a. 4p.

SOUZA, E.B.; MELO JÚNIOR, J.C.F.; ANDRADE, I.G.V.; SILVA, A.R. Probabilidade de ocorrência da evapotranspiração de referência $\left(\mathrm{ET}_{0}\right)$ para o município de Petrolina, PE. In: Congresso Brasileiro de Engenharia Agrícola, 43, 2014, Campo Grande. Anais... Campo Grande, 2014. 4 p.

VELLAME， L.M.; QUEIROZ, M.Z.S.S.; OLIVEIRA, A.S. Probabilidade de ocorrência da evapotranspiração de referência em Ouricuri-PE. In: I Inovagri International Meeting \& IV Workshop Internacional de Inovações Tecnológicas na Irrigação, 2012, Fortaleza. Anais... Fortaleza, 2012. 5p. 